\title{
Strengthening the Postgraduate programme in Community Medicine: a Step towards Advancing Reforms in Public Health Education in India.
}

\author{
Rajguru, C. ${ }^{1}$, Sankhe, L. $^{2}$, Akarte, S.V. ${ }^{3}$, Sharma, A. ${ }^{4}$ \& Zodpey, $S .{ }^{5}$
}

\begin{abstract}
Introduction: Public Health Education in India is at Crossroads is a well dwelled fact. The huge difference between the demand from health services and the inability of the medical education system to fulfil it, is creating a problematic situation.

Objectives: This study was conducted with the objective of exploring the need for change in the community medicine postgraduate course as felt by the academicians, public health experts as well as the postgraduate students. Through this study an attempt was made to identify the key changes needed in the course with a broader objective of restructuring the community medicine course to bring it at par with the required standards.
\end{abstract}

Methods: The compiled findings from extensive literature search, opinion of public health experts in the field, and faculty member teaching community medicine and postgraduate student's views suggests a complete revamping of the course contents with focus of skill based training.

Results and conclusion: The course should be implemented, so as to impart skills which will give maximum practical exposure to the students with community involvement. The experience from private non-governmental organisations will also widen their vision of public health. The importance of research should be emphasized and there is a need to improve the quality of research in medical colleges. In a nutshell, the course should evolve so as to develop the community medicine postgraduate student into an academician, administrator and researcher. This agenda if taken further on various technical panels will certainly assist in creating the required public health workforce.

Key words: Curriculum, Skills, public health specialist.

\section{Introduction}

The Health scenario of the world is rapidly transforming due to globalisation, epidemiological and demographic transitions, evolving technology and fluctuating economic trends.

\footnotetext{
${ }^{1}$ Associate Professor

${ }^{2}$ Associate Professor

${ }^{3}$ Professor \& Head

Department of Community Medicine, Grant Medical College, Mumbai, India.

${ }^{4}$ Program Officer, Public Health Foundation of India ${ }^{5}$ Vice-President, Public Health Foundation of India

Corresponding Author:

Dr. Lalit Sankhe, Associate Professor,

Department of Community Medicine, Grant Medical

College, Mumbai, India.

E-mail:lalit.sankhe@gmail.com
}

These transformations are creating huge demands on the health system. The public health system is constantly analysing and trying to meet the challenges. The public health professionals play a key role in these circumstances. The human resource in public health is scarce; there is an urgency to meet the demand of the public health professional of the nation.

Traditionally the public health specialists in the country have been produced by the departments of community medicine in medical colleges and specialised institutes such as the All India Institute of Hygiene and Public Health (Kolkata) and a few others. The role of departments of community medicine in imparting Public Health Education has been recognised from ages. Though community 
medicine and public health are considered at par with each other, there are certain distinct differences in the scope, training and application of both (Patro et al., 2011). The objective and goal of postgraduate medical education in community medicine are to produce competent specialist to manage the teaching departments in the medical colleges, or to manage health services and national health programs or to conduct biomedical research in the discipline of community medicine. (Negandhi et al., 2010)

Currently the postgraduate programme in community medicine is implemented through community medicine departments of medical colleges. The subject of community medicine is taught in the undergraduate as well as postgraduate curriculum. The postgraduate course of community medicine is a three year full time residential course with six terms and completion of a thesis/ dissertation. The certification of the course is after acceptance of thesis and passing the university final examinations at the end of three years.

In India, 224 Medical colleges offer postgraduate courses in community medicine and 45 colleges offer a diploma course, and every year 771 postgraduates and 101 diploma holders in community medicine are produced. The smallness of the number is sufficient to point towards the fact that there is a need to strengthen the community medicine course with respect to increasing the numbers of postgraduates being produced yearly as well as the quality and content of the course (Medical Council of India, New Delhi, 2015).

The Medical Council of India provides guidelines to conduct the Postgraduate courses in community medicine, thereby leaving implementation to the respective departments and institutes. This leads to loss of uniformity and standardisation, affecting the quality of the course. The quality of teaching has not been appropriate to cater to needs of the healthcare delivery system in India (Garg \& Gupta, 2011).

This exercise is to address the above issue and make an attempt so as to produce competent public health specialists in the future, enhancing the existing skills and competencies. The aim being to evaluate the structure of the curriculum based on the current and future public health needs and provide evidence based recommendations to officials and decision makers for planning and developing a comprehensive, evolutionary, futuristic model so as to elevate community medicine course in the medical colleges and bring it on par with national and international standards.

\section{Methods}

In a quest to improve the quality of postgraduate education in community medicine and thereby contribute to the ever demanding public health workforce, the following systematic process (a mixed method approach) was followed to gain relevant information.

1. Literature Review (Identification of skills and competencies for post graduate course of community medicine): An extensive literature search was conducted through search engines like Google, Google scholar, I seek, PubMed to name a few. The key words for the literature search were Public Health, Community Medicine, Curriculum, postgraduate program, India. The articles found were then filtered according to the relevance of the subject and finally 36 articles were referred for the current study. The observations of the authors were compiled as supportive evidence.

2. Expert Consultation: Communications with 17 public health experts (academicians, researchers, administrators in the field of public health both from the government as well as non-government organisations) discussing the study subject, over a period of one year, yielded valuable information regarding need of public health professionals in various organisations, the changing role of public health professionals and the skills required to fulfil the increasing demands.

3. Assessing the current implementation and expectations of the current postgraduate students of community medicine course: A survey questionnaire was designed on consultation with the subject experts. The response was sought by emailing the questionnaire to present postgraduate (PG) students of community medicine from randomly selected medical colleges from all over India. A total 53 students responded. The questionnaire consisted of two parts; (1) implementation of the course in their institute at present, (2) students expectations from this course and their perception about the usefulness of the current program to prepare them to face the public health workforce demands. This gave an insight into the current practices of implementation of the PG course in the 
respective medical colleges as well as expectations of students about the course.

4. Consultative Meet: A consultative meet of the faculty members of community medicine, purposively a mix of mid-level and senior faculties as well as representatives from the medical sciences university was conducted. The consultative meet was attended by 46 participants. The meet was organised in sessions where in the first session the current practices of the need for reforms, detailed analysis of current post graduate curriculum, was discussed. The community medicine course discussion was divided into following four domains - Theory, practical, research and Assessment. The participants were randomly divided into four groups each addressing the issues of one of the above domains. Each group had a facilitator and a recorder from the organising institute. Each group discussed the domains at length and finally the recommendations from each group were presented to all the participants for further inputs. The outcome of the consultative meet was in the form of recommendations to the university.

\section{Results}

\section{Literature review}

The extensive literature review conducted for the study included reports from 1910 (Flexner report) the Welch-Rose report, various international and national authors study findings to the latest Lancet commission and lancet series on Medical Education. It also included findings from discussion forums on the issue of bringing about changes in the medical education and also public health education. The findings on shows that since more than 100 years the medical education as a whole has been evolving from time to time and trying to meet the challenges posed by the disease scenario of the world. At the beginning of the 21st century, the medical education has not been able to keep pace with the challenges presented by new infectious diseases, environmental and behavioural risks, and the rapid epidemiological and demographic transition (Frenk et al., 2010).

The disease scenario is changing rapidly, but comparatively the public health education is at a standstill. The findings suggest the need for reforms, demand for such a change is palpable through the review of various forums, also the need for a complete revamp public health education specifically community medicine which produces public health professionals in the country is strongly felt (Frenk et al., 2010; WHO, 2006; WHO, 2008 ).

The public health education has received great attention as shown by a series of research papers: The research papers conclude that there is a presence of a smouldering situation about to erupt due to the mismatch of professional competencies to the health demands of the population, because of obsolete, stagnant course curriculum.

The Research papers, question the quality of Public Health professionals produced in the country. The papers focus on the need for reforms in the community medicine course so as to improve the quality of public health education in the country, the processes and the reforms needed to be adopted at all levels. The research papers also focus on the vital role of community medicine departments in the medical institutions, in changing the public health education scenario of the country.

The research papers deliberate on the need for skill based or competency based education, with patient centred, community oriented, evidence based, using new information technologies, multidisciplinary approach with exposure to policy, law, economics, sociology, leadership (Page \& Baranchuk, 2010; Sommer, 1915; Suresh, 2012; Kumar, 2013; Negandhi et al., 2012; Sunder, 2004; Sharma \& Zodpey, 2010).

The public health experts having diverse experience from across the country expressed that to deal with the evolving public health challenges; there is an urgent need to address the issue of scarce human resources in public health sector. Along with quantity, quality also matters. The skills and competencies of the public health workforce is a critical matter and cannot be further ignored.

The public health experts felt that the postgraduate students of community medicine, lack the skills and expertise demanded by the job profile of a public health specialist in national as well as international agencies. There is a definite gap between the theoretical and practical aspects of teaching, and the content of the teaching is not based on the futuristic needs of the health system.

Community exposure should be the key component of the course. Introducing the 
students to the functioning of National and international agency by giving them practical experience will certainly strengthen their skills as public health specialist. Along with the mandatory course content, guidance to acquire newer skills should be provided. Acquiring soft skills like communication, presentation, documentation should be focused on.

The survey questionnaire result reflects the feedback received from the current post graduate students of the randomly selected medical colleges all over India. On compilation of the overall observations, the comprehensive impression gained was that,

- The students were unaware about the exact skills that are required by a public health specialist.

- Although the postgraduate academic activities were held regularly in most of the colleges and term wise timetables were prepared, it was not in sync with the $\mathrm{MCl}$ guidelines. There was variation in the implementation of the programme, with each college following their own way of teaching.

- The postgraduate activity schedule has been the same since ages and has not been revised to meet the current expectations of the course.

- The postgraduate activities consist of departmental academic activities like journal article presentations, seminars, book reviews, case presentations. Other than the departmental activities the post graduate students are posted in urban health centre and rural health centre for a period of 1 to 3 months respectively. In addition to it they are also posted in the clinical departments of medicine, obstetrics and gynaecology, paediatrics, chest and $\mathrm{TB}$, and a few more with some variation from college to college. The period of postings varies from college to college and the $\mathrm{MCl}$ guidelines are not strictly followed.

- The role of the postgraduate students in the above postings is that of a Resident Medical Officer, and gives very restricted practical exposure.

- The theoretical lectures are conducted in the department mostly by the department faculties, the frequency is in the range of 1 2 lectures per month. Guest lectures by subject experts are organised less frequently/ rarely. There is no fixed schedule for conducting lectures.

- The practical component of the course includes microbiology practicals (conducted in few departments, not all) and field visits to areas of public health importance, there is no set pattern for these field visits and the schedule is not strictly adhered to. In many instances the field visits are not in sync with the places of public health importance.

- The involvement in undergraduate teaching is very restricted and unsupervised.

- The post graduate research being an important component of the post graduate course is not revised since many years. There are no guidelines to be followed from the inception to the completion of the thesis work. The completion of the thesis is decided by the post graduate guide. There is no checklist, based on which the thesis is declared to be completed. The involvement of the postgraduate students in other departmental research projects (if any) is very minimal.

- The postgraduate student is not regularly and frequently assessed for his/her performance. There are no term ending examinations conducted, nor are the Post graduate activities like journal article, seminar or other presentations evaluated and graded. In all, the student are not assessed or evaluated by the department for the entire three years for performance. There is no weightage in terms of marks given to any of the activities that a post graduate student is supposed to do during his/her course period.

- Almost all the participants commented that the PG course in community medicine is not of much help in preparing them to face the public health challenges with confidence.

\section{Consultative meet}

The presentation from the representatives of the community medicine departments of participating medical colleges reflected the findings from the survey questionnaire to a greater extent. The situation of all the colleges was more or less the same. Lack of term wise guidelines, community and clinical exposure during postings, training of the students in the art of teaching, soft skills were a few issues 
identified. As per the group, there was no set pattern of implementing the course. There was no provision for any national or international agency exposure. In some institutes the assessment was in the form of written examination conducted yearly, but the grades were not considered for their final assessment. The opinion of all the participants was that this exercise to revise the curriculum and further modification to enhance the community medicine post graduate course was the need of the hour. The following were the suggestions of the group:

The entire syllabus should be divided into terms that would spread over a period of 3 years, each term consisting of 6 months.

\section{1) Theory Domain}

It was recommended that PG program should start with an induction training of 2 weeks which would explain the role of community medicine specialist in health/ lectures by health administrators so as to sensitise the students to the vastness of public health, also it should be used to familiarise the students with the institute, various departments and the urban and rural community served by the institute. There should be guest lectures from the public health specialist working in different domains. The group felt the need for formulating a manual which would explain the course objectives and the term wise curriculum provide guidelines to proceed throughout the course and also tell them what is expected from them at the end of each term as well as at the completion of the course.

Theory topics should be reviewed and upgraded according to the future scenario. To address the issue of gap in the knowledge of the students as well as faculties regarding the health care delivery system and the actual implementation of the programmes, the coordination between the health services and medical education departments should be improved.

The concept of e-learning should be introduced in the curriculum. Students should be encouraged to participate in seminars, conferences, workshops, guest lectures, Student debates and IT demonstrations within the institute as well as the national and international level.

It was unanimously felt that there were certain topics which were very important as far as public health was concerned and needed emphasis in the postgraduate course: health economics, information education communication, behavioural change communication, health planning, writing project protocol, advanced biostatistics, demography and family welfare, school health, international health, health policies and program.

Along with the theoretical lectures and supplementary readings, the problem solving capacity of the students should be developed. The course should have self- assessing, review exercises at the end of each major topic. There should be student faculty discussions on case studies and different health scenarios. Access to standard national and international books, free and paid indexed, national and international journal, guidelines of various national health programmes etc. should be made available to the students. They should be trained in writing research proposals, reports so as to enhance their documentation skills.

\section{2) Practical Domain}

Based on the group's recommendations, practical exposure to the Post graduate students should be structured in a way so as to acquire the required skills and exposure, mainly the hands on training wherever applicable.

An exhaustive list of places of public health importance along with the guidelines for every visit should be prepared. The visits should be evaluated by exercises related to the visits. The community exposure part of the public health course, as a public health specialist exposure to the Community is a must. This exposure should be in the form of community based surveys organised by the department or conducting community based research as part of their dissertation. Also post graduate students should be encouraged to participate in the community based activities like health education campaigns etc. of the departments on a regular basis.

The clinical postings should be based on clearly defined objectives and guidelines, so as to develop the community physician skills of the students. The students should be posted in specialised clinics like TB, Leprosy, HIV, Diabetic clinic etc., so as to sensitize them to the need of the priority health issues and the current practices followed in the same areas.

Epidemic investigation skills can be developed by exposing them to real life situations if any, or give them assignments based on it. 
Acquiring administration skills is an important part of being a public health specialist, the students should be posted in departments like, MRD , Medical and Surgical Stores, Medical Control Room etc. to get to know the functioning of a health care unit / institute. The students should be well aware of all the ethical issues, accreditation guidelines, and various acts and laws related to hospital administration. The health care delivery system is undergoing a sea of change. The students should be well versed with the health care delivery system and exposed to the implementation of various health programs from the state to the village level. To acquire this they should be posted to the Health service department at different levels.

Looking at the future needs the students should be trained to polish their communication skills-oratory, counselling as well as presentation skills. They should be trained in the art of teaching thus enhancing pedagogical skills

3) Research Domain

It was universally felt by the group that the aim of postgraduate research should be for the students to acquire a spirit of scientific inquiry and should be oriented to the principles of research methodology and epidemiology.

It has been cited that $85 \%$ of all research work done is wasteful or inefficient, with deficiencies in the relevance of research question for clinicians or patients of local area or not addressing the local needs, inappropriate design and methods, inaccessibility of the full report, and issues about the work being unbiased and clinically meaningful (Agarwal et al., 2013; Calmers \& Glasziou, 2009).

The research component was proposed to be organised term wise as shown in Table 1.

Table 1: Term-wise organization of the research component

\begin{tabular}{|c|c|c|}
\hline Terms & Components & Activities \\
\hline \multirow[t]{2}{*}{ First } & Thesis & $\begin{array}{l}\text { Selection of Topics of Interest ( } 5 \text { topics) along with competent } \\
\text { ROL (to know research done up to date). }\end{array}$ \\
\hline & Training Program & Research Methodology \\
\hline \multirow{3}{*}{ Second } & Thesis & Pilot study, Submission of Thesis Protocol \\
\hline & Training Program & Biostatistics, Protocol Writing \\
\hline & Conference & Participation with or without paper presentation \\
\hline \multirow{4}{*}{$\begin{array}{l}\text { Third } \\
\text { and } \\
\text { Fourth }\end{array}$} & Thesis & $\begin{array}{l}\text { Data Collection; Thesis Update; Blind data validation by faculty } \\
\text { members other than guide }\end{array}$ \\
\hline & Training Program & Communication skill, Protocol and Manuscript writing \\
\hline & $\begin{array}{l}\text { Conference } \\
\text { (state/national) }\end{array}$ & $\begin{array}{l}\text { Compulsory paper presentation; Paper writing for presented } \\
\text { conference paper and submission to indexed journal }\end{array}$ \\
\hline & Journal Club & $\begin{array}{l}\text { Critical appraisal of Published Research Articles (Surprise } \\
\text { Appraisal) }\end{array}$ \\
\hline \multirow[t]{2}{*}{ Fifth } & Thesis & $\begin{array}{l}\text { Data collection and analysis; thesis update; writing and submission } \\
\text { to University }\end{array}$ \\
\hline & Journal Club & $\begin{array}{l}\text { Critical appraisal of Published Research Articles (Surprise } \\
\text { Appraisal) }\end{array}$ \\
\hline Sixth & Thesis & Research Meet (Inter-college thesis presentation) \\
\hline
\end{tabular}

Attending conferences and training programmes should be compulsory. Weightage in the final assessment should be given to attending a conference, presenting paper in the conference, this will motivate them to undertake short research activities and thereby help them to develop their skills in the research domain. It should be mandatory for every student, to write a paper based on the thesis and keep it ready for publication before the submission of thesis. 


\section{4) Assessment Domain}

Any programme, be it educational, should be regularly monitored and frequently assessed to maintain the quality and achieve the objectives of the program to its maximum.

It was proposed by the group that the current system of assessment at the end of the course based on theory and practical examination does not provide a view of the students' performance over the course period of two/ three years.

In addition to the final university examination, $50 \%$ weightage should be given to the performance of the student throughout the course and this should be periodically assessed. Each presentation, seminar, teaching activity should have points and weightage in the final assessment.

Periodic examinations should be conducted at the end of each term with a fixed syllabus in the form of written or oral examinations. Periodical weekly or fortnightly exercises should be given to the students to be completed and submitted within a specified deadline, with points for each exercise.

The above mentioned skills assessment should be done and weightage allotted to it. Timely completion of work, quality of work, reactiveness and special achievements should be given extra weightage / points.

A well balanced assessment framework should be developed so as to assess the overall performance of the students.

\section{Discussion and conclusion}

Human resource issues in public health and quality of public health professionals produced urges us to consider revising and transforming the postgraduate course so as to create a cadre of professional who can function as educators , public health specialists, researchers, policy makers and implementers, public health managers and leaders .

From Flexner's report in 1910, to Welch-Rose report in 1915, the need for change in medical education as a whole and public health has been stressed on. Long back in 1910, Abraham Flexner presented a report, expressing the need for revamping the entire medical education, his report brought in accreditation, centralization of medical training led to high quality of medical education. Thus Flexner's report inspired a turning point in medical education (Page \& Baranchuk, 2010).

This was followed by Welch-Rose report in 1915 , the purpose of which was "development of the spirit of investigation and the advancement of knowledge". As stated by Elizabeth Bee, the Welch-Rose report is a blueprint for development of professional schools of public health in United States. It was a step forward in public health education (Sommer, 1915).

The uncertainty raised over production of public health experts in India was cited in Rajeev Garg et al publication with a stress upon the need for a paradigm shift in Community medicine in medical colleges wherein the author enlists the current educational course in community medicine and dwells upon whether we in India are producing public health experts (Garg \& Gupta, 2011).

Authors in various publications over a period of time have been presenting the history and evolution of public health education in India, the role of Community Medicine departments of medical colleges in shaping the future of public health education, the needs for reforms and the strategies for improving public health education in India (Suresh, 2012; Kumar, 2013; Negandhi et al., 2012; Sunder, 2004; Sharma \& Zodpey, 2011; Baral et al., 2010, Sharma \& Zodpey, 2010).

The postgraduate course in community medicine should be looked at from a broader perspective encompassing a varied array of disciplines. The skills and competencies of the course should be identified timely and absorbed into the course curriculum. A similar study has clearly analysed the processes and needs of postgraduate public health education and suggested some solutions for institutions to adapt or adopt so as to improve the quality of public health education (Suresh, 2012).

Research studies have time and again suggested the need for change in the public health education regarding the curriculum as well as the actual implementation of the program with emphasis on renewing the content considering the future public health needs (Sharma \& Zodpey, 2011; Baral et al., 2010; Sharma \& Zodpey, 2010).

National Rural Health Mission also emphasizes the need to change the face of public health education by promoting various educational 
programs in different aspects of public health to strengthen the public health workforce.

This exercise thus delivers comprehensive technical and very detailed inputs regarding the strategies to strengthen postgraduate education in community medicine:

- Induction training of the postgraduate students in community medicine.

- Revamping the entire syllabus and segregation of the topics for each term.

- The curriculum should focus on enhancing problem solving capacity of the students and community based.

- Developing a symbiotic relationship with the public health system and national and international agencies for expanding the vision of the students.

- Encouraging authentic and effective scientific research

- Faculty development programs and encouraging the faculties to acquire and enhance newer skills is a must by promoting collaborations with national and international universities and strict monitoring should be inherent component of this program.

- Collaborations with community organisations should be initiated.

- Curriculum reforms should be outcomebased and monitored by continuous assessment, it should also increase the capacity to integrate knowledge and transform it into practical application

- Development of a culture of critical inquiry, to prepare physicians with a renewed sense of socially responsible professionalism.

\section{Way forward}

The entire public health community throughout the nation is vibrating with the notion of revolutionary transformation in the field of public health education.

To improve the health of our community and to face the challenges in the future we need well qualified public health Professionals who will deliver quality health services to our community as well as conduct research to further improve the health status and also create future public health specialist of high calibre.

This attempt to revolutionalise the public health education should be well represented before the higher authorities and substantial efforts should be taken to pursue the issue of public health education reforms. Similar opportunity should be exploited to sensitise the professionals in the field of public health towards the need for reforms and obtain their valuable inputs on the same.

A national level working group should take up the task of pursuing the matter further. Political will is a must for such endeavours, securing it will confirm the execution of the proposition. The demand thus should be created and met at all levels with the broader futuristic vision of providing high quality of public health professional to the country in the coming years.

\section{References}

Agarwal, J., Kulkarni, M., Mohan, U. \& Das, V. (2013) IndiaClen recommendations for improving postgraduate medical research and publication research and publication, Clinical Epidemiology \& Global Health, 1, 2, pp. 53-84.

Baral, N., Paudel, B.H., Das, B.K., Aryal, M., Gautam, A. \& Lamsal, M. (2010) Preparing tutors for problem-based learning: An Experience from B. P. Koirala Institute of Health Sciences, Nepal, Kathmandu University Medical Journal, 8, 29, pp. 141-145.

Calmers, I. \& Glasziou, P. (2009) Avoidable waste in the production and reporting of research evidence, Lancet, 374, 9683, pp. 86-89.

Garg, R. \& Gupta, S. (2011) Are We Really Producing Public Health Experts in India? Need for a Paradigm Shift in Postgraduate Teaching in Community Medicine, Indian Journal of Community Medicine, 36, 2, pp. 93-97.

Frenk, J., Chen, L., Bhutta, Z.A., Cohen, J., Crisp, N., Evans, T. \& Fineberg, H. (2010) Health professionals for a new century: transforming education to strengthen health systems in an interdependent world, Lancet, 376, 9756, pp. 1923-1958.

Kumar, R. (2013) Healthcare and medical education reforms in India: What lies ahead? Journal of Family Medicine and Primary Care, 2, 2, pp. 123-127.

Medical Council of India, New Delhi: Vision (2015) [Online] Available at: https://old.mciindia.org /tools/announcement/MCl_booklet.pdf (Accessed 15th April 2014). 
Negandhi, H., Sharma, K. \& Zodpey, S. (2012) History and Evolution of Public Health Education in India, Indian Journal of Public Health, 56, 1, pp. 12-16.

Negandhi, H., Sharma, K. \& Zodpey, S.P. (2010) How Can Departments of Community Medicine Shape the Future of Public Health Education in India? Indian Journal of Public Health, 54, 4, pp.184-189.

Page, D. \& Baranchuk, A. (2010) Flexner Report: 100 years later, International Journal of Medical Education, 1, pp. 74-75.

Patro, B.K. \& Singh, A. (2011) Community Medicine Departments and Public Health Education in India at Crossroads, Indian Journal of Public Health, 55, 1, pp. 56-57.

Sharma, K. \& Zodpey, S. (2010) Need and Opportunities for Health Management Education in India, Indian Journal of Public Health, 54, 2, pp. 84-91.

Sharma, K. \& Zodpey, S. (2011) Public Health Education in India: Need and Demand Paradox, Indian Journal of Community Medicine, 36, 3, pp. 178-181.
Sommer, A. (1915) The Welch-Rose Report- A Public Health Classic, Delta Omega Honorary Public Health Society, [Online] Available at: http://s3.amazonaws.com/aspph-wp-production /app/uploads/2014/02/The-Welch-Rose Report.pdf

Sunder L. (2004) Scenario of Postgraduate Medical Education in Community Medicine in India [Online] Available at: https://pdfs.semantic scholar.org/195d/247581026ed7676e04a9765 468d60976c97c.pdf [Accessed April 2014].

Suresh, K. (2012) Influencing Public Health without Authority, Indian Journal of Public Health, 56, 1, pp. 22-30.

WHO, World Health Organization (2006) The World Health Report: working together for health, Geneva: World Health organization.

WHO, World Health Organization (2008) Scaling up, saving lives, Task for Scaling Up Education and Training for Health Workers, Global Health Workforce Alliance, Geneva: World Health Organisation. 